\title{
Correction \\ Correction: Chang et al. Decursinol Angelate Arrest Melanoma Cell Proliferation by Initiating Cell Death and Tumor Shrinkage via Induction of Apoptosis. Int. J. Mol. Sci. 2021, 22, 4096
}

\author{
Sukkum Ngullie Chang ${ }^{1,2}$, Imran Khan ${ }^{2,3}$, Chang Geon Kim ${ }^{1}$, Seon Min Park ${ }^{1}$, Dong Kyu Choi ${ }^{4}$, \\ Heejin Lee ${ }^{4}\left(\mathbb{D}\right.$, Buyng Su Hwang ${ }^{5}\left(\mathbb{D}\right.$, Sun Chul Kang ${ }^{2, *}$ and Jae Gyu Park ${ }^{1, *(D)}$
}

Citation: Chang, S.N.; Khan, I.; Kim, C.G.; Park, S.M.; Choi, D.K.; Lee, H.; Hwang, B.S.; Kang, S.C.; Park, J.G. Correction: Chang et al. Decursinol Angelate Arrest Melanoma Cell Proliferation by Initiating Cell Death and Tumor Shrinkage via Induction of Apoptosis. Int. J. Mol. Sci. 2021, 22, 4096. Int. J. Mol. Sci. 2022, 23, 1629. https://doi.org/10.3390/

\section{ijms23031629}

Received: 26 December 2021

Accepted: 17 January 2022

Published: 31 January 2022

Publisher's Note: MDPI stays neutral with regard to jurisdictional claims in published maps and institutional affiliations.

Copyright: () 2022 by the authors Licensee MDPI, Basel, Switzerland. This article is an open access article distributed under the terms and conditions of the Creative Commons Attribution (CC BY) license (https:// creativecommons.org/licenses/by/ $4.0 /)$.
1 Advanced Bio Convergence Center (ABCC), Pohang Technopark Foundation, Pohang 37668, Korea; sukkumchang@gmail.com (S.N.C.); rjs6538@naver.com (C.G.K.); seonmin@ptp.or.kr (S.M.P.)

2 Department of Biotechnology, Daegu University, Gyeongsan 38453, Korea; imranakhan7@gmail.com

3 The Hormel Institute, University of Minnesota, Austin, MN 55912, USA

4 New Drug Development Center, DGMIF, 88 Dongnae-ro, Dong-gu, Daegu 41061, Korea; dongkyu@dgmif.re.kr (D.K.C.); jini150117@gmail.com (H.L.)

5 Nakdonggang National Institute of Biological Resources, Sangju 37242, Korea; hwang1531@nnibr.re.kr

* Correspondence: sckang@daegu.ac.kr (S.C.K.); jaegpark@gmail.com (J.G.P.);

Fax: +82-53-850-6569 (S.C.K.); +82-54-223-2780 (J.G.P.)

The author wishes to make the following correction to this paper [1]:

In the original publication, there was a mistake in Figure 2. During manuscript preparation, the Figure $2 \mathrm{~B}$ CDK4 blot was erroneously duplicated with CDK2. The authors did minor changes in statistical analysis in corresponding western blot quantification. These changes do not affect the conclusion and findings of the research article. The corrected Figure 2 and legend appears below.
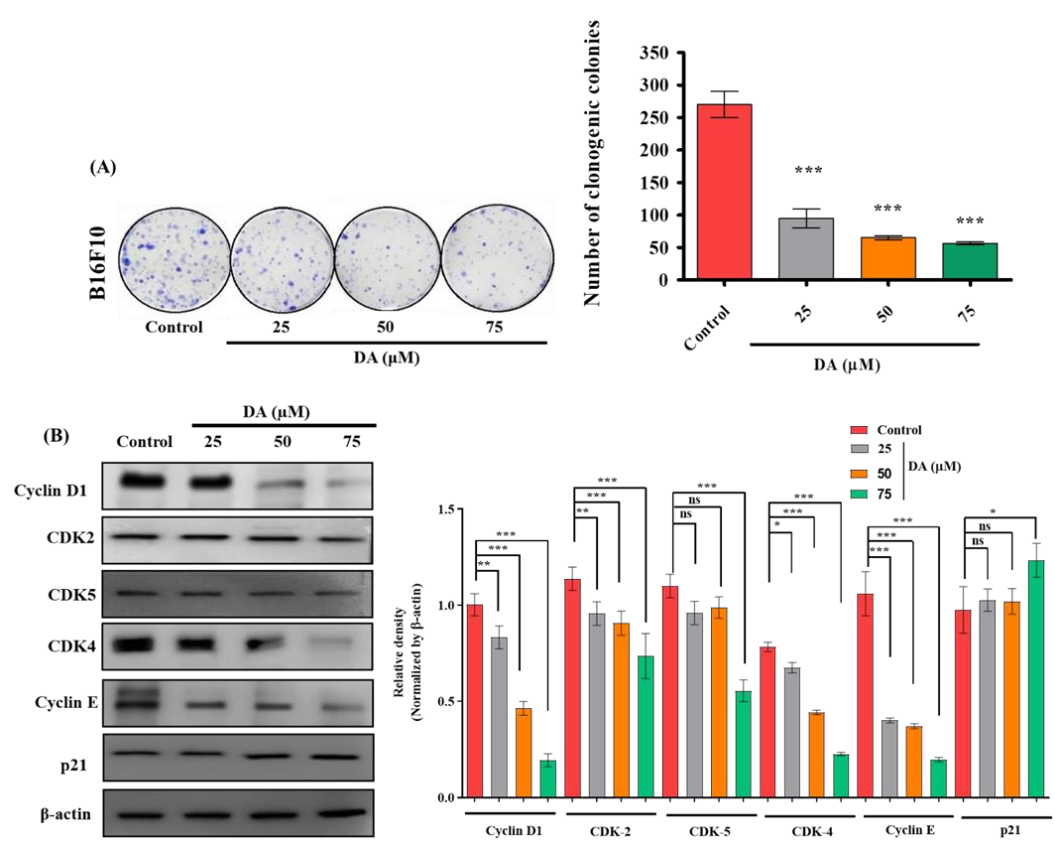

Figure 2. (A) Clonogenic assay and quantification of B16F10 cells cultured in the presence and absence of DA over 7 days, followed by crystal violet staining. (B) DA was treated to B16F10 cells for $24 \mathrm{~h}$ and cell cycle protein levels were detected, such as cyclin D1, CDK2, 5, 4, cyclin E, and p21. Densitometry analysis of the respective proteins was evaluated by Image J software, and results were normalized with $\beta$-actin. The data are represented as the means \pm standard deviation (SD) of three independent experiments; ns-non-significant; ${ }^{*} p<0.05,{ }^{* *} p<0.01,{ }^{* * *} p<0.001$ vs. control, calculated through ANOVA prism. 
The authors apologize for any inconvenience caused and state that the scientific conclusions are unaffected. The original publication has also been updated.

\section{Reference}

1. Chang, S.N.; Khan, I.; Kim, C.G.; Park, S.M.; Choi, D.K.; Lee, H.; Hwang, B.S.; Kang, S.C.; Park, J.G. Decursinol Angelate Arrest Melanoma Cell Proliferation by Initiating Cell Death and Tumor Shrinkage via Induction of Apoptosis. Int. J. Mol. Sci. 2021, 22, 4096. [CrossRef] [PubMed] 